\title{
Mediated Deliberation
}

EIKE MARK RINKE

University of Mannheim, Germany

Deliberation is a form of discussion in which an individual or a group carefully examines a problem and considers proposals for solution that reflect a variety of perspectives with the aim of arriving at a well-reasoned solution. Mediated deliberation is the subtype of such discussion undertaken by means of mediated communication. Mediated deliberation was introduced by Benjamin I. Page who defined it as "discussions among government officials, experts, and professional commentators, which are conveyed to a broader audience through the mass media of communications" (1995, p. 245). Contemporary understandings extend beyond this focus on the transmission of political elite discussion and encompass any discussion enabled by media communication, including citizens as independent actors. Mass-mediated deliberation is the most normatively and empirically important type of mediated deliberation in modern large-scale societies, since-although it does not generally allow interaction between "senders" and audiences - it is the form of communication with the greatest potential for the political inclusion and engagement of citizens. Mediated deliberation through mass communication is a process whereby newspapers, online news outlets, and other media help the wider public understand and think through issues in at least a quasi-deliberative way. "Deliberativeness" is a gradual quality of any communication. It denotes the degree to which a communicative act, or series of acts, conforms to a catalog of deliberation principles like respecting all those affected by policies, the responsive exchange of "good" reasons, and so on (see below for a more detailed catalog). As a deliberation subtype, mediated deliberation has strong normative connotations and implies assumptions about the collective responsibilities of media producers and users. Although researchers have focused on mediated deliberation in public affairs media, the concept extends seamlessly to cultural media such as literary works, movies, and entertainment television.

\section{Mediated deliberation in deliberative democratic theory}

Deliberative democratic theory has paid relatively little attention to mediated deliberation. Paradoxically, given the importance of mediated modes of communication in modern societies, a focus on small-group nonmediated deliberation has prevailed in this literature. This development is due to a shift in interest moving towards questions of democratic deliberation rather than deliberative democracy on a broad scale

The International Encyclopedia of Political Communication, First Edition. Edited by Gianpietro Mazzoleni. (c) 2015 John Wiley \& Sons, Inc. Published 2015 by John Wiley \& Sons, Inc.

DOI: 10.1002/9781118541555.wbiepc189 
(Chambers, 2009). Some deliberative theorists have even pushed against an expansive concept of deliberation that would encompass various forms of political discussion. According to their argument, discussion should only qualify as deliberation if it meets requirements so strict as to almost necessarily exclude most forms of mediated communication from the deliberation domain (e.g., general public-spiritedness of participants and equal participation).

The neglect or even outright dismissal of mediated communication in deliberative democratic theory is rooted in more or less explicit assumptions about the mass media. Various modern works in deliberative theory chide them for what is seen as excessive exclusiveness and selectivity regarding their input and a lack of responsive exchanges in mediated communication. The role of mass media in public deliberation has often been addressed in discussions of how to grant deliberative mini-publics more societal impact. This particular route to engaging with mediated communication has led some authors to conclude that media are generally unfit to promote deliberative democracy, not only due to remediable failures on their part but also due to their essential characteristics. The general reservations against mediated communication in deliberative theory have also included concerns about their tendency to promote a passive mode of citizen engagement with politics and a fragmented and asymmetric style of communication that is seen as fundamentally opposed to the engaging, coherent, and reciprocal kind of communication aspired to in normative accounts of deliberation.

The dismissal of mediated communication as a relevant component of deliberative democracy is surprising since it belies the fact that some of the most important classical influences on deliberative democratic theory have come from writers who were also theorists of the public sphere. A prominent example is Jürgen Habermas, who wrote extensively on the role of media in a deliberative democracy. Émile Durkheim, another classical influence on deliberative theory, discussed the epistemic-cognitive value of mediated public deliberation in his lectures on morals and law; and John Dewey added the social-moral values it may realize. Though often neglected in classical-modern deliberative democratic theory, echoes of these classical contributions continue to be heard, for example in the works of James Bohman (e.g., 2000).

Engagements with the role of mediated deliberation have recently regained traction with a theoretical movement towards a systemic conception of deliberative democracy that includes the media infrastructure of large-scale societies (Parkinson \& Mansbridge, 2012). This new engagement with the role of media in public deliberation pushes research towards more explicit accounts of that role and has opened up opportunities for the integration of face-to-face and mediated forms of communication in a common

deliberative framework. A pioneer in the development of such a framework was the late Bernhard Peters (2008); a pioneer in the realm of empirical application is Rousiley C. M. Maia (2012).

\section{Components of mediated deliberation}

The emerging systems perspective in normative deliberative democratic theory has led to a growing recognition by researchers that public spheres are multiple and differ in 
their scale (i.e., degree of mediation) and orientation towards political or civic life (i.e., type of content) (Breese, 2011). A proper theory of mediated deliberation needs to disaggregate the concept of the public sphere into its constituent components (e.g., levels of analysis and/or stages of the communication process).

The "systemic turn" in deliberative democratic theory also suggests that proper models of deliberation need to assess the applicability of deliberative principles in different societal and communicative contexts. The development of context-sensitive models implies a need to renegotiate deliberative principles to conceptualize deliberation under varying suboptimal speech conditions. Fully developed context-specific normative theories of mediated deliberation are not yet available although some scholars have identified specific contributions that particular media forums (e.g., television news) can make to the wider deliberative system. One important aspect of this trend towards disaggregation and contextualization of mediated deliberation is the identification of the specific resources that media offer to the public exchange of opinions and reasons over and above those furnished by face-to-face communication.

Mediated deliberation may be studied from two general perspectives. The first is the external, system perspective. Here, mediated deliberation is viewed as deliberation across a media system and within the frame of a "division of deliberative labor" in the system. The key question is: How deliberative is the media system as a whole? Some theoretical statements employing this perspective have been published (e.g., Habermas, 2006), but they remain rare and relatively vague.

The second is the internal, forum perspective. It focuses on mediated deliberation as deliberation occurring in particular media forums that together form the system of society-wide public deliberation. This perspective is more established and has been more widely employed in empirical research. Its key question is: How deliberative are individual media types, formats, or programs? A forum of mediated deliberation is made up of three components: the arena in which actors communicate publicly; the gallery of an active audience observing them; and the backstage in which public communicators (e.g., journalists and politicians) prepare and interact removed from public view (Ferree, Gamson, Gerhards, \& Rucht, 2002). Most studies of mediated deliberation are studies of the production, content, and consequences of communication in specific media forums such as newspapers, online discussion boards, or radio talk shows.

The forum perspective points to a distinctive feature of mediated deliberation: its triadic structure in which discourse participants talk to one another while addressing the audience in the gallery as well as media producers (the "forum hosts") in an effort to promote their own positions. In such a communication situation a competitive political system makes substantive policy consensus among actors in the arena highly unlikely. This feature of mediated deliberation is a consequence of its publicness and the "triple contingency" of mass-mediated communication which, unlike much face-to-face communication, is influenced by the third point of view brought to the situation by the observing public. This recognition has led researchers to an alternative normative criterion for judging the success of mediated deliberation: the production of internal reasoned dissent (Wessler, 2008). According to this criterion, actors in the public arena need not come to substantive agreement about the resolution of contested issues. The public display of civil disagreement should rather increase the number of publicly 
available ideas, opinions, and arguments, clarify different aspects of and perspectives on issues, discredit untenable arguments, and increase the recognition of different points of view. The enlargement of the public argument pool through mediated deliberation is expected to increase the quality of citizens' decision-making. It is also expected to improve policy outcomes by imposing on political elites the requirement to justify their policy proposals in public. The promotion of mutual understanding and recognition of opposed points of view is expected to facilitate political compromise at the elite level and its acceptance at the level of the public.

Although the reasoned dissent criterion constitutes theoretical progress over earlier formulations of normative demands towards communication in the mediated public sphere, it remains a central task of contemporary mediated deliberation research to specify pragmatic (or "realistic") ideals for deliberation in different media spaces.

\section{Production of mediated deliberation}

Mediated deliberation is coproduced by all actors involved in any of its specific instances. Investigations working within a deliberation framework have concentrated on two actor types: journalists responsible for crafting mediated deliberation in public affairs coverage, and deliberative event practitioners aiming to maximize the impact of their deliberative process in the wider public sphere. Much less is known about the deliberative orientations of officials in the public sphere. Although mediated deliberation theory expects officials normatively and empirically to change their communication styles depending on the characteristics of the social settings they operate in, empirical evidence of such effects is in short supply.

Research on the production of mediated deliberation has focused on journalism. This is not a coincidence, since political journalism can be reconstructed as the primary social institution rooted in the normative ideal of a deliberative public sphere (Peters, 2008). For deliberative democratic theory the presence of public argumentation based on justification and reason-giving is the centerpiece of a deliberative public sphere. News journalism has been conceived of as a central institution of public justification, most directly expressed in understandings of journalism as reason-giving journalism and news as justificatory news (Ettema, 2007). Journalism creates communicative spaces in which the discursive power of justification can come to bear and legitimate binding political decisions.

Production studies investigate whether the justificatory function of journalism in mediated deliberation is reflected in journalists' ideals of the public sphere, their general professional ideologies, and their specific attitudes towards the goals and purposes of deliberation.

The canon of journalism ethics includes a general professional ideal of "keeping people informed." This ideal has a deliberative connotation to it because the provision of high-quality political information will help citizens in understanding the merits of public arguments for or against specific policies. However, beyond such generally agreed professional ideals, journalists may vary more in their views on what an ideal public sphere should look like. Whether they espouse more elite-oriented, citizen-oriented, or discourse-oriented views of the public sphere influences professional behaviors such as 
their relations to political actors like officials, citizens, and intellectuals. More generally, it may also influence their professional ideologies and their concomitant propensity to engage in announcement or investigative journalism (elite centered), advocacy journalism (citizen centered), or public/civic journalism (discourse centered).

From a mediated deliberation perspective, each of these approaches to journalism may contribute differently to a functional public political discourse. However, the public journalism project is most closely related to concerns about the quality of mediated deliberation. This project originated in the United States and promotes a form of journalism that focuses on enabling informed citizen deliberation about important community problems. Public journalism highlights the Socratic role of journalists to give voice to those who would not otherwise be heard in the public sphere: ordinary citizens, especially those from socially marginalized groups. It also promotes a journalism that is oriented towards substantive solutions to social problems (Rosen, 1999).

Forms of public journalism have been implemented at about one-fifth of US newspapers, most of them published by small news organizations. Much less public journalism has been practiced in other forums such as television or radio. While journalists in the United States generally hold positive attitudes towards public journalism, they endorse those public journalism practices the most that deviate least from conventional elitecentered forms of journalism. The most discourse-centered public journalism practices like organizing forums for citizen deliberation and cooperating with civic organizations are generally met with greater skepticism, even among public journalists themselves. Such reservations towards public journalism are relevant for mediated deliberation because discourse-centered journalism produces news content that is richer in substantive and mobilizing political information, which in turn increases audiences' interest in and knowledge of community issues as well as their inclination to become politically active.

A more concrete influence on the production of mediated deliberation than their models of "good journalism" is found in journalists' approaches to events designed to generate deliberative political engagement of citizens. Journalists appear to be generally sympathetic to efforts at increasing citizen deliberation and believe in their positive effects on citizen knowledge. However, they are more pessimistic about the potential of deliberative initiatives to have legislative impact. These attitudes towards concrete forms of public deliberation are another facet of the professional discourse ethics and civic-mindedness of journalists likely to impact their production of mediated deliberation.

The finding that many journalists largely approve of deliberation-friendly professional norms has inspired research into why they often violate these norms in their daily work. One way in which journalists sometimes retreat from their deliberative responsibilities is by adopting a pessimistic view of citizens' ability to contribute to political discourse. This view appears to offset the more sanguine perspective described above. Another way in which journalists may rationalize failures to live up to their deliberative principles is by lowering expectations towards their own role as contributors to deliberative discourse in a "realist" idiom if that appears more opportune than actually enacting these principles. 
Deliberative event practitioners are also relevant to the production of mediated deliberation. One task of deliberative event organizers is to work out effective communication strategies that produce "mediated meta-deliberation" about their events and increase their public impact. Practitioners can enhance the impact of their event on mediated deliberation if they provide journalists with clear outcome-related information such as lists of policy recommendations resulting from the deliberative process. They may also increase the news value of their deliberative process by pointing out contentious issues deliberated on in their event without sacrificing a focus on its deliberative principles.

Another aspect of mediated deliberation production concerns the consequences of online environments for deliberative qualities of discussion. Online deliberation encompasses a wide range of mediated deliberation processes. The quality of such processes typically benefits from the presence of explicit discussion rules and moderators/facilitators prompting online discussion participants to adopt a reflective approach to topics under discussion.

\section{Content of mediated deliberation}

Mediated deliberation encompasses the entirety of political topics and issues discussed in mediated public forums and it provides a framework for assessing the democratic value of specific communication content. The main question here is about the deliberative qualities of certain types or instances of such content.

What should the content of ideal mediated deliberation look like? Quite generally, good mediated deliberation constitutes a process within which civil contestation leads, at a minimum, to the emergence of reasoned public dissent. This process includes rebuttals between representatives of divergent ideas and reciprocal substantive criticism of actors in the public sphere. Ideal mediated deliberation therefore includes a diverse set of speakers, enables substantive responses between them, and features explicit justifications for political opinions. The applicability of other criteria of deliberation like civility and potential reversals of opinion in light of good reasons varies more between media forums and stages of the political process. Uncivil political communication, for example, may be more admissible in the early agenda-building stages of the political process, in which social grievances are brought to public attention, than during substantive deliberation stages in which solutions for public problems are discussed.

Although normative criteria for the content of mediated deliberation are applied differently across different media forums, a general catalog encompasses the following six items. Collectively, they constitute necessary conditions for reasoned dissent to come about in a media forum:

1 Inclusiveness of actors. Mediated deliberation should include voices from all corners of society ("multiperspectivalness"). The broadest and most commonly applied distinction between actor types is that between speakers from the political center (including representatives of formal-institutional politics) and the political periphery (including representatives of civil society). 
2 Ideational inclusiveness. Mediated deliberation should include different ideas regarding public problems (i.e., topics) and solutions. Though this criterion is intimately connected to agentic accounts of inclusiveness (cf. criterion 1) it is a distinct task of journalism to filter and aggregate relevant public proposals.

3 Responsiveness. Mediated deliberation should include substantive responses between diverging ideas regarding public problems and solutions, carried out either directly through their representatives or indirectly through professional intermediaries like journalists or mediators.

4 Justification. Mediated deliberation should include substantive reasons and arguments that justify specific claims and opinions about public problems and solutions rather than mere opinions or pronouncements of positions. Public justification is central to the instrumental (problem-solving) as well as the moral (recognitionpromoting) quality of public discourse.

5 Civility. Mediated deliberation should be characterized by civil communicative exchanges between political actors. Though some tolerance of incivility is necessary for a deliberative public sphere, civil mutual respect allows citizens to honestly consider the merits of all sides' arguments and accept the general legitimacy of political opposition.

6 Meta-deliberation. Mediated deliberation should include substantive exchanges about the proper values, norms, and rules of public discourse. Such metadeliberation is supposed to correct violations of deliberative norms in discursive practice and generate the continuous discursive-democratic innovation. This criterion includes journalistic self-coverage and general discussions of the rules and norms of public debate but also the mediated presentation of deliberative participation events.

This catalog of general criteria needs to be adapted for different types of forums for mediated deliberation. Journalism continues to produce the central forums for mediated deliberation, such as public affairs news or political talk shows. But nonjournalistic forms of mediated deliberation have gained political importance with the establishment of the Internet as a relevant space for public political discussion. The systems perspective on mediated deliberation suggests that different forums may fulfill distinct deliberative functions in different societies, highlighting the importance of comparative and context-sensitive mediated deliberation research (Downey, Mihelj, \& König, 2012).

Three types of research on the content of mediated deliberation can be distinguished. The first type consists of research operationalizing key concepts of deliberative democratic theory for use as empirical indicators of discourse quality. From such research, various catalogs of normative criteria for assessing mediated deliberation have been developed (see Wessler \& Rinke, 2014).

The second type of research on the content of mediated deliberation consists in a concept-based translation of work that in its original presentation is not explicitly related to deliberative democratic theory but that is still relevant to one or more components of mediated deliberation. This translation is done through conceptual normative assessment that examines a given communication concept for its 
logical implications with regard to deliberative values (see Rinke, Wessler, Löb, \& Weinmann, 2013). Such examination involves considering the attributes included in the concept (its so-called intension) and assessing their relevance to mediated deliberation. If they are relevant, conceptual normative assessment determines if the mere presence of the communication phenomena made observable by the concept promotes, hampers, or is ambivalent for mediated deliberation. For example, one common way in which journalists cover election campaigns is by contrasting candidates' diverging points of view on a policy issue. Such depiction of a campaign as a competition of ideas implies some measure of reciprocal responsiveness between those ideas. This journalistic device therefore has an inherent deliberative value to it. The relation between the mere presence of the phenomenon made observable by a concept and the quality of mediated deliberation so identified is deterministic.

The third type of research on the content of mediated deliberation is focused on making evidence-based connections between mediated deliberation indicators of the kind developed by the first type of content-based research and other nondeliberative indicators of media performance. This type of research engages in empirical normative assessment that examines the empirical relations of a given phenomenon (such as the kind of media system or a certain type of communication) with communication phenomena that are normatively relevant to mediated deliberation. The empirical deliberative value of a given communication phenomenon depends on the degree to which it is causally related to positive or negative indicators of deliberative performance (e.g., the degree of opinion justification or civility). For example, the deliberative value of journalists' tendency to depict electoral contests as a strategic game of self-interested politicians tends to be low because it creates a news discourse that is less inclusive, less civil, and less based on substantive reasons (Rinke et al., 2013). The relation between the empirical relations of a given phenomenon and the quality of mediated deliberation is probabilistic.

Factors on multiple analytical levels influence the deliberativeness of public affairs news and other media content. Research shows that distinct national news cultures and political systems produce television news that is much more deliberative in established than in defective democracies. News also tends to be more deliberative in powersharing political systems than in power-concentrating systems (Wessler \& Rinke, 2014).

The market orientation of media organizations is a factor that has a particularly strong bearing on the deliberativeness of their news output. Public service channels, nonpartisan programs, and in-depth news shows usually produce more deliberative public affairs content than their respective counterparts. This tendency is particularly pronounced with respect to the "throughput" of mediated deliberation (i.e., opposing positions, responsive utterances, justification, civility) but also concerns the input side of mediated deliberation (i.e., its actor inclusiveness and multiperspectivalness). The average deliberative advantage of public service channels is grounded in their specific statutory and legal responsibilities, their professional culture of providing "high-quality" political debate, and often their relative lack of economic pressures to abandon such legal and professional commitments. 
Specific features of political media content that are not themselves components of deliberation also influence its deliberativeness. Partisan reporting, for example, tends to decrease the deliberativeness of news content. First, the diversity of speakers on the news is lowered by journalists' tendency to privilege sources that support their own point of view (so-called "opportune witnesses": Hagen, 1993). Second, the diversity of relevant topics, ideas, and facts can also get lowered in much the same way (so-called "instrumental actualization": Kepplinger, Brosius, \& Staab, 1991).

News journalism also shows trends toward greater negativity, scandal orientation, sensationalism, soft news focus, and sound-bite journalism. Each of these developments, in its own way, is also relevant to the content of mediated deliberation.

The increasing negativity of journalistic reporting may promote substantive criticism and responsive argumentation about political issues, and an increasing focus on political scandal in news reporting may provide opportunities for public discussion of morally relevant issues and actions of political actors. However, the contemporary market-driven trends in many media systems towards more sensationalist reporting can undermine these opportunities for rational argumentation and responsiveness by promoting a focus on vivid stories and argumentative exaggeration as a journalistic device.

Whether soft news and a focus on personalities rather than collective political actors (e.g., parties) divert journalistic attention from ideas and argumentative, reason-based discourse per se remains an open question. Although these trends are much maligned, it may rather be an excessive focus on politicians' private lives ("privatization") that crowds out the discussion of political issues and ideas needed for successful mediated deliberation. Media personalization per se would become anti-deliberative in its consequences primarily if it led to a depiction of political difference as being personal rather than philosophical, but whether that is happening is an as yet open empirical question.

Another global trend in television news reporting, the tendency to use ever shorter segments of uninterrupted speech for speakers on the news (i.e., sound-bite journalism), diminishes the deliberative value of news by decreasing the frequency with which speakers actively justify their political positions instead of just declaring them (Rinke, 2013).

While sound-bite journalism harms mediated deliberation, the evidence is less clear on how actor inclusiveness affects other aspects of deliberation. Featuring civil society representatives in news coverage has been shown to increase the occurrence of uncivil acts in coverage in the context of a specific "hot-button issue" like abortion (Ferree et al., 2002) but does not seem to lower the deliberative quality of routine television news coverage (Rinke, 2013). Featuring academic experts, on the other hand, improves the ideational inclusiveness, multiperspectivalness, and nuance, and leads to more rebuttal acts (van der Wurff, Verhoeven, \& Gadellaa, 2013).

In addition, the format choices editors and journalists make when producing the news not only are matters of taste but also impinge on the quality of mediated deliberation that can be expected from it. Dialogical news elements (e.g., interview segments and substantive responses among speakers) can promote mediated deliberation by providing opportunities for speakers to give and demand from each other substantive 
reasons for their substantive political positions (Rinke, 2013). Finally, the different frames journalists may use to report on political actors and events also influence the deliberative quality of news content (see examples of the strategic game and contestation frames above).

\section{Consequences of mediated deliberation}

Mediated deliberation is not an end in itself. Normative theory expects mediated deliberation to improve the political process by exerting influence on both citizens and politicians.

Vis-à-vis citizens, reasoned dissent in mediated deliberation is expected to provide models of deliberative behavior, at least as far as dispositions necessary for the emergence of reasoned dissent are concerned. Mediated deliberation is not necessarily expected in modern normative theory to showcase communication behavior oriented towards understanding, although such citizen behavior still is assumed to be important. Mediated deliberation theory instead suggests that resources other than mediated public deliberation are more promising in this regard, first among them institutions of formal education or event-based deliberative mini-publics. But citizens are expected to learn from the rich pool of arguments about important issues of public concern presented to them in mediated deliberation and attach greater value to deliberative discussion as a mode of outcome-oriented political communication. Exposure to mediated deliberation of public affairs also is expected to add to the legitimacy of authoritative decisions both in the abstract and in the eyes of citizens.

Vis-à-vis politicians, mediated deliberation is expected to lead to more rational, effective policy outcomes not only by holding public officials accountable for their authoritative actions - the approach to public political communication favored by liberal theories of democracy - but also by putting on them discursive pressure to work out policy proposals that are justifiable through recourse to convincing reasons (Wessler, 2008).

Overall, empirical tests of these expectations in normative theory are rare. One of the main difficulties for mediated deliberation effects research is to disaggregate mediated deliberation into its constituent components and develop middle-range theories of their specific effects. Such a theory would explain, for example, how the presence or absence of justifications for political positions affects citizens' opinion formation.

More is known about the consequences of the mediation component of mediated deliberation. The impersonal nature of mass media exposes citizens to political positions opposing their own more often than do everyday face-to-face communications. Such exposure to disagreement through mediated deliberation is in line with the emphasis on discursive plurality in deliberative democratic theory and so contributes to the realization of broader deliberative ideals (Mutz \& Martin, 2001).

But the consequences of mediation reach further. Audiences may, for example, be more inclined to accept "better reasons" to adopt a certain position in the anonymous, noninteractive setting of mass-mediated deliberation: The relative lack of identity maintenance and social categorization pressures in the impersonal communication situation of receiving mass-mediated messages reduces incentives for citizens to maintain opinion consistency (as opposed to being perceived as "flip-flopping" on issues). This 
particular deliberative benefit of mediated deliberation is consistent with a model of communication effects that sees messages as potentially affecting their senders by foreclosing internal deliberation processes (Pingree, 2007). An audience member in massmediated deliberation may on average pay less attention to reasons she is exposed to than a participant in face-to-face deliberation, but since she does not generally become a "sender" and contribute her own opinion to the mediated discussion she may be more circumspect in forming an opinion and more open to changing her mind in light of new reasons encountered through mediated deliberation.

Effects of mediated deliberation on individuals also depend on their discursive preferences. Though mediated deliberation may influence these preferences to some extent, its effectiveness depends on the patterns of discursive dispositions in citizens that collectively form a political discourse culture. Citizens appear to generally value discourse participants that act in line with the norms of good deliberative discourse formulated in deliberative democratic theory. They tend to value public argumentation that is reasoned, is principled, and evidences a focus on certain core issues; a general open-mindedness of discourse participants; and a visible commitment to continuing deliberation until public problems are resolved (Jørgensen, Kock, \& Rørbech, 1998). However, the appreciation of discourse norms in the abstract and in individual opinion formation processes may be at odds with simultaneous entertainment motivations in the citizen audience. For example, people tend to be more entertained by uncivil, combative forms of discussion in political talk shows. This may make them more likely to tune in, but such mediated discussion may also lower their political trust and willingness to accept political points of view different from their own as legitimate.

Citizen expectations towards mediated deliberation also vary across types of actors. In the United States, citizens appear to discount elected officials as productive participants in public discourse (Evans, 2012). This may lower the chances for mediated deliberation involving public officials to gain traction in the population and suggests as ideal more citizen-oriented forms of mediated deliberation like talk shows involving ordinary citizens as guests or televised town hall meetings.

Finally, expectations of citizens influence the effects of mediated deliberation in another way: When they anticipate their own opinion to become important, in a voting decision or before consequential interaction about an issue, citizens show greater interest in using mediated deliberation for their own opinion formation process.

\section{Future directions in researching mediated deliberation}

Mediated deliberation provides a relatively recent framework for engaging with mediated political communication. The production, content, and consequences of mediated deliberation are bound to become more relevant in contemporary media societies, both theoretically and practically. However, mediated deliberation remains less developed conceptually and researched empirically than face-to-face deliberation processes. Future research may improve on the status quo in terms of both its general theoretical foundations and the three specific components of mediated deliberation (production, content, and consequences). 
Regarding theoretical foundations, the consequences of mediation for each dimension of the deliberation process need to be more thoroughly understood. What, for example, are the consequences of publicness for the various participants in mediated deliberation processes? Answering this question will help understand the implications of the modes of social integration in media societies for democratic politics. A more focused engagement with the consequences of mediation will also further establish mediated deliberation as a research domain of its own.

Another challenge will be to connect mediated deliberation to the systemic turn in deliberative democratic theory. Conceptually, this undertaking can benefit from a greater integration of insights from cognitive social and discursive learning theory (e.g., Strydom, 2007) and, fundamental theoretical disagreements notwithstanding, poststructuralist discourse analysis (e.g., Van Dijk, 1988). These developed macrosocial approaches to public discourse may contribute to a more holistic systems perspective on mediated deliberation.

Empirically, three needs suggest future steps to better account for the role of mediated deliberation in deliberative systems. First, there is a need to focus more on the development of context-sensitive normative and empirical theories so as to characterize the varying contributions of different forms of mediated deliberation in different deliberative systems. This will involve greater efforts to harness the epistemic and reformist potential of cross-national comparative work to identify how different features of political and media systems influence mediated deliberation. Comparative work can provide evidence needed to inform media reform aimed at improving deliberative practice in national public spheres. Research in nondemocratic societies could help in understanding how deliberation norms and principles may be used not only for virtuous goals of improving democracy but also to stage "façade mediated deliberation" that fuses features of authoritarian rule like an unchecked decision-making authority of political leaders with deliberative principles and procedures like the civil exchange of reasons for and against a policy while selectively eschewing others (e.g., agentic inclusiveness or the contestation of claims made by power holders).

Second, there is a need to investigate the evolution of deliberative systems in which mediated deliberation may react to the configuration of the overall system. One example is the development of a specific kind of political discourse in the medium of comedy or satire in reaction to recurrent violations of deliberative principles in environments characterized by highly strategic, professional, and adversarial political communication.

Third, there is a need to consider more systematically the role of narrative forms of mediated deliberation in deliberative systems. Many forms of mediated political communication such as movies, novels, and experiential accounts of social problems by ordinary citizens have a narrative rather than an argumentative structure. Yet they may be consequential for the quality of the overall deliberative system and possibly carry unique deliberative potentials such as increasing access to public discourse for citizens who are less willing or able to participate in the principled argumentative discussion of issues. 
Regarding the production of mediated deliberation, a major challenge will be to identify which societal conditions - cultural, legal, and organizational-are most conducive to the emergence of successful mediated deliberation. While recent research shows that consensus democracies and majoritarian democracies differ in the styles of mediated deliberation they produce, a persisting puzzle in this domain is whether their institutional designs promote higher quality mediated deliberation in some cases and worse quality in others. Another open question is whether political actors adopt more deliberative communication practices in more deliberative public spheres, as normative theory expects. Tracking politicians' political communication behavior in different forums could answer this question.

Regarding the content of mediated deliberation, one important challenge for future research will be to build theory by addressing more systematically the internal consistency of normative models of mediated deliberation: Do different components of mediated deliberation support each other in "virtuous deliberative circles" or may they also restrain each other in "deliberative trade-offs"? Is a responsive justification behavior of political actors, for example, most likely to occur in some mediated contexts when expectations towards all-around civility are lowered?

Regarding the consequences of mediated deliberation, research will have to bridge the divide between mediated and unmediated forms of public deliberation and investigate their interplay more thoroughly. Open questions here concern the effects of how citizens' and politicians' use of mediated deliberation depends on their individual contexts (e.g., will they actually need to participate in political conversation, discussion, or even deliberation at some point?) and the deliberative system at large (e.g., do features of political systems like the place of referendums in decision-making influence people's engagement with mediated deliberation?). An integrated perspective on mediated and unmediated deliberation will foreground the deliberative benefits and shortcomings of mass-mediated deliberation relative to interpersonal deliberation as well as their complementarities as distinct components of deliberative systems.

SEE ALSO: Civility; Deliberation; Deliberative Polls; Digital Public Sphere; Legitimacy, Democratic; Media Democracy; Media Performance; Normative Theories; Public Journalism Movement; Public Sphere

\section{References}

Bohman, J. (2000). The division of labor in democratic discourse: Media, experts, and deliberative democracy. In S. Chambers \& A. N. Costain (Eds.), Deliberation, democracy, and the media (pp. 47 -64). Lanham, MD: Rowman \& Littlefield.

Breese, E. B. (2011). Mapping the variety of public spheres. Communication Theory, 21(2), 130 - 149. doi: 10.1111/j.1468-2885.2011.01379.x

Chambers, S. (2009). Rhetoric and the public sphere: Has deliberative democracy abandoned mass democracy? Political Theory, 37(3), 323-350. doi: 10.1177/0090591709332336

Downey, J., Mihelj, S., \& König, T. (2012). Comparing public spheres: Normative models and empirical measurements. European Journal of Communication, 27(4), 337-353. doi: $10.1177 / 0267323112459447$ 
Ettema, J. S. (2007). Journalism as reason-giving: Deliberative democracy, institutional accountability, and the news media's mission. Political Communication, 24(2), 143-160. doi: $10.1080 / 10584600701312860$

Evans, M. S. (2012). Who wants a deliberative public sphere? Sociological Forum, 27(4), 872-895. doi: 10.1111/j.1573-7861.2012.01360.x

Ferree, M. M., Gamson, W. A., Gerhards, J., \& Rucht, D. (2002). Shaping abortion discourse: Democracy and the public sphere in Germany and the United States. Cambridge, UK: Cambridge University Press.

Habermas, J. (2006). Political communication in media society: Does democracy still enjoy an epistemic dimension? The impact of normative theory on empirical research. Communication Theory, 16(4), 411 -426. doi: 10.1111/j.1468-2885.2006.00280.x

Hagen, L. M. (1993). Opportune witnesses: An analysis of balance in the selection of sources and arguments in the leading German newspapers' coverage of the census issue. European Journal of Communication, 8(3), 317 - 343. doi: 10.1177/0267323193008003004

Jørgensen, C., Kock, C., \& Rørbech, L. (1998). Rhetoric that shifts votes: An exploratory study of persuasion in issue-oriented public debates. Political Communication, 15(3), 283-299. doi: $10.1080 / 105846098198902$

Kepplinger, H. M., Brosius, H.-B., \& Staab, J. R. (1991). Instrumental actualization: A theory of mediated conflicts. European Journal of Communication, 6(3), 263-290. doi: $10.1177 / 0267323191006003002$

Maia, R. C. M. (2012). Deliberation, the media, and political talk. New York, NY: Hampton.

Mutz, D. C., \& Martin, P. S. (2001). Facilitating communication across lines of political difference: The role of mass media. American Political Science Review, 95(1), 97 - 114.

Page, B. I. (1995). Speedy deliberation: Rejecting “1960s programs” as causes of the Los Angeles Riots. Political Communication, 12(3), 245-261. doi: 10.1080/10584609.1995.9963072

Parkinson, J., \& Mansbridge, J. (Eds.). (2012). Deliberative systems: Deliberative democracy at the large scale. Cambridge, UK: Cambridge University Press.

Peters, B. (2008). Public deliberation and public culture: The writings of Bernhard Peters, 1993-2005 (H. Wessler, Ed.; K. Tribe, Trans.). Basingstoke, UK: Palgrave Macmillan.

Pingree, R. J. (2007). How messages affect their senders: A more general model of message effects and implications for deliberation. Communication Theory, 17(4), 439-461. doi: 10.1111/j.1468-2885.2007.00306.x

Rinke, E. M. (2013). Justificatory news: Investigating the contextual antecedents of justification in the news (doctoral dissertation). University of Mannheim, Germany.

Rinke, E. M., Wessler, H., Löb, C., \& Weinmann, C. (2013). Deliberative qualities of generic news frames: Assessing the democratic value of strategic game and contestation framing in election campaign coverage. Political Communication, 30(3), 474-494. doi: $10.1080 / 10584609.2012 .737432$

Rosen, J. (1999). What are journalists for? New Haven, CT: Yale University Press.

Strydom, P. (2007). A cartography of contemporary cognitive social theory. European Journal of Social Theory, 10(3), 339-356. doi: 10.1177/1368431007080699

Van der Wurff, R., Verhoeven, P., \& Gadellaa, M. (2013). Scientists and deliberativeness of European public television news. Journal of Science Communication, 12(3), A02.

Van Dijk, T. A. (1988). News as discourse. Hillsdale, NJ: Erlbaum.

Wessler, H. (2008). Investigating deliberativeness comparatively. Political Communication, 25(1), 1-22. doi: 10.1080/10584600701807752

Wessler, H., \& Rinke, E. M. (2014). Deliberative performance of television news in three types of democracy: Insights from the United States, Germany, and Russia. Journal of Communication, 64(5), 827-851. doi: 10.1111/jcom.12115 
Eike Mark Rinke is a research associate in the Mannheim Centre for European Social Research (MZES) at the University of Mannheim, Germany. He studies normative aspects of political communication with a particular interest in public deliberation in cross-national perspective. 